\title{
Adult rhabdomyosarcoma of the gall bladder: case report and review of published works
}

\author{
T M Al-Jaberi, N Al-Masri, A Tbukhi
}

\begin{abstract}
A 90 year old woman with alveolar rhabdomyosarcoma of the gall bladder is described. The patient died with tumour metastasis four months after diagnosis despite complete excision of the tumour. The histological, immunohistochemical, and ultrastructural features of the tumour are summarised and published works are reviewed.
\end{abstract}

(Gut 1994; 35: 854-856)

The hepatobiliary system is an extremely rare site for the primary lesion of rhabdomyosarcoma. To our knowledge, rhabdomyosarcoma has been described in the gall bladder of three adult patients, ${ }^{1-3}$ as reported in English language publications. Two of the cases were of the embryonal type,,$^{13}$ and one mixed embryonal and alveolar type. ${ }^{2}$

In this report we present a fourth case of rhabdomyosarcoma of the gall bladder in an adult and the first case of the pure alveolar type. The histological features, immunohistochemical findings, and ultrastructure of the tumour are also described.

\section{Department of General Surgery T M Al-Jaberi}

and Department of Pathology, Jordan University of Science and Technology and Princess Basma Teaching Hospital, Irbid, Jordan N Al-Masri

\section{Department of} Pathology, University of Texas, Galveston A Tbukhi

Correspondence to: Mr I M Al-Jaberi, 38 Clarendon Road, Leeds LS2 9PX.

Accepted for publication 20 October 1993

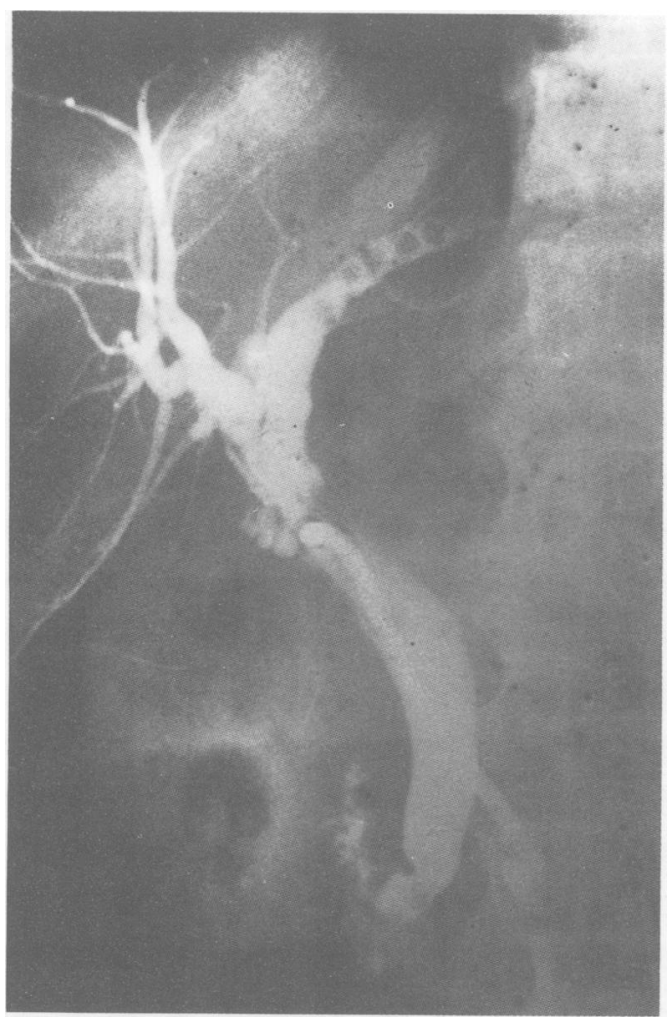

Figure 1: T tube cholangiogram showing retained stones in the left hepatic and the common bile ducts.

\section{Case report}

A 90 year old woman was admitted to Princess Basma hospital in June 1991 with a two year history of right upper quadrant pain, which became continuous over the last month and associated with loss of weight and appetite. There was no history of jaundice. On examination she was found to have low grade fever, right upper quadrant tenderness, and a mass. Laboratory investigations showed: packed cell volume $29 \%$, white blood cell count 12800 , and slightly increased bilirubin and liver enzyme activities. Ultrasound showed a gall bladder mass and multiple gall stones.

At laparotomy, a gall bladder mass $10 \times 15$ $\mathrm{cm}$ in diameter was completely excised. The common bile duct was found dilated and was explored, multiple common bile duct stones where removed, and a $\mathrm{T}$ tube was inserted. The liver seemed normal. A T tube cholangiogram showed retained common bile duct stones including some in the left hepatic duct (Fig 1). By the time endoscopic rectrograde cholangiopancreatography was done, only one common bile duct stone remained and was extracted at the same time as sphincterotomy. Clinical investigations showed no evidence of a primary tumour in the genital system or in other sites in which rhabdomyosarcoma is known to occur as a primary tumour.

Two months later, the patient started to have recurrence of the right upper quadrant pain and progressive deterioration of her general health. One month later she presented with an enlarged left supraclavicular lymph node. Excisional biopsy was done. There was no further intervention upon the patient's request apart from analgesics until her death four months after her initial presentation. A necropsy was not performed.

\section{Methods}

Microscopic sections of formalin fixed tissue were prepared and stained with haematoxylin and eosin. For immunoperoxidase stains, 4 $\mu \mathrm{m}$ sections from paraffin wax embedded tissues were prepared using the avidin-biotin complex technique. ${ }^{4}$ The antibodies used included: cytokeratin (AE 1,3; Hybritech, Indianapolis, IN), carcinoembryonic antigen, epithelial membrane antigen, desmin and vimentin (Dako, Santa Barbara, CA), myosin and myoglobin (Biogenex, Dublin, CA), and muscle specific actin (Enzo, New York, NY). The chromogen used in this study was diaminobenzidine (Sigma, St Louis, MO). Representative sections of formalin fixed tissue were taken for electron microscopic examination. 
Review of published reports

\begin{tabular}{llllll}
\hline \multirow{2}{*}{$\begin{array}{l}\text { Case } \\
\text { report }\end{array}$} & Author/year & \multicolumn{2}{l}{ Patient } & & \\
\cline { 2 - 4 } & Age & Sex & Histology & Survival \\
\hline 1 & Edmonson $^{1} 1967$ & 80 & $\mathrm{~F}$ & Embryonal & Unknown \\
2 & Yasuma and Yanaka $^{2} 1971$ & 67 & $\mathrm{~F}$ & Embryonal and alveolar & 45 Days \\
3 & Willen and Willen $^{3} 1982$ & 68 & $\mathrm{~F}$ & Embryonal & 2 Months \\
4 & Present case 1992 & 90 & $\mathrm{~F}$ & Alveolar & 4 Months \\
\hline
\end{tabular}

\section{Pathological findings}

Macroscopic findings - a tumour mass $10 \times 9 \times 7.5 \mathrm{~cm}$ at greatest dimension was seen arising from the posterior wall of the gall bladder. Extensive necrosis and haemorrhage were present in the central regions of the tumour. The gall bladder was filled with stones.

Microscopic and immunohistochemical findings - as Figure 2 shows, the gall bladder epithelium seems intact over the tumour. Tumour cells were round polygonal cells with a high nuclear to cytoplasmic ratio and prominent nucleoli. The cytoplasm was eosinophilic and granular, however, and cross striations were not seen. The tumour cells formed nests surrounded by fibromuscular septa reminiscent of the solid variant of alveolar rhabdomyosarcoma. The results of immunohistological tests showed positive staining of tumour cells for vimentin and desmin; the other muscle markers - actin, myosin, and myoglobin - were negative. Cytokeratin, epithelial membrane antigen, and carcinoembryonic antigen were negative.

Ultrastructural examination showed tumour cells that lacked epithelial characteristics such as cell junctions, desmosomes, villi, and gland formation. No thin filaments, thick filaments or $\mathrm{Z}$ bands were seen.

\section{Discussion}

Carcinoma is the commonest malignant tumour of the gall bladder, while primary sarcoma of the gall bladder is a rare
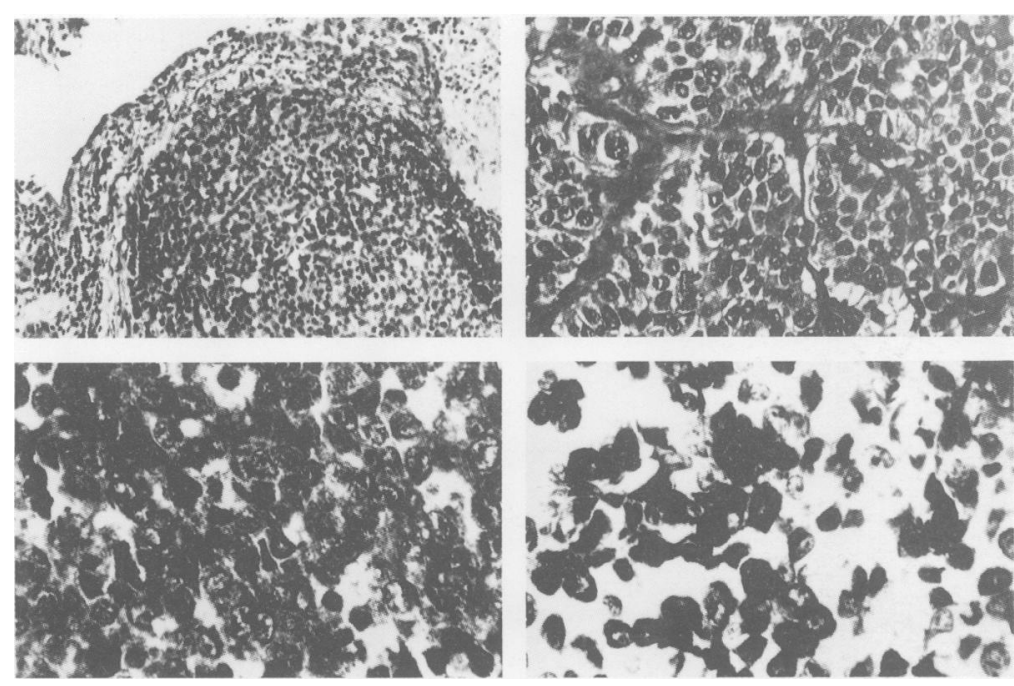

Figure 2: Rhabdomyosarcoma cells seen beneath an intact gall bladder mucosa (upper left; haematoxylin and eosin $\times 100)$. Tumour cells form nests surrounded by fibrovascular septa, solid alveolar pattern (upper right; haematoxylin and eosin $\times 400$ ). Positive desmin and vimentin staining of tumour cells in lower left and lower right pictures respectively (avidin-biotin complex technique $\times 400$ ). occurrence. ${ }^{23}$ In adults, rhabdomyosarcoma of the hepatobiliary system is extremely rare..$^{23} \mathrm{~A}$ review of English language published works showed three cases of adult gall bladder rhabdomyosarcoma, ${ }^{1-3}$ two of them of the embryonal type ${ }^{13}$ and one of the mixed embryonal and alveolar type (Table). ${ }^{2}$ These three cases and this case report have been reported in women over 65 years. Jaundice has been reported in two of the cases, ${ }^{23}$ but jaundice was not found in our patient despite the presence of multiple common bile duct stones. One of the previous cases (patient number 3) had a right upper quadrant mass similar to our patient ${ }^{3}$ while another (patient number 2) was similar to our patient in having a left supraclavicular lymph node metastasis. ${ }^{2}$ The longest survival was four months after the onset of symptoms (as reported in our patient) despite what seemed to be complete excision. All four patients had gall stones. ${ }^{1-3}$ Our patient, however, was the only one having common bile duct and hepatic duct stones. Gall stones have been reported to occur in about $80 \%$ of all sarcomas of the gall bladder. ${ }^{23} \mathrm{~A}$ similar case of gall bladder alveolar rhabdomyosarcoma have been reported in French published works, ${ }^{5}$ and one case of alveolar rhabdomyosarcoma have been reported in the common bile duct. ${ }^{6}$

In the paediatric age group, rhabdomyosarcoma of the biliary tree should be considered in the differential diagnosis of obstructive jaundice after choledochal cyst. ${ }^{7}$ It is usually of the embryonal (sarcoma botryoides) variety. ${ }^{7-13}$ Since the first reported case in 1875 by Wilks and Moxon, ${ }^{14} 52$ cases of hepatobiliary rhabdomyosarcoma in the paediatric age group have been reported in the world publications by $1986 . .^{615}$ Forty five of these cases were in the biliary tree ${ }^{15}$ and one in the ampulla of Vater. ${ }^{16} \mathrm{~A}$ longer survival period has been reported recently, even in the presence of extensive tumour, in those patients given aggressive surgical treatment followed by radiation and chemotherapy. ${ }^{9}$

Although the histology of this tumour is that of a poorly differentiated malignancy, the positive vimentin staining in association with negative expression of epithelial markers (keratin, epithelial membrane antigen, and carcinoembryonic antigen) support the diagnosis of a mesenchymal tumour. The histological picture and positive desmin staining (Fig 2) confirm the diagnosis of alveolar rhabdomyosarcoma. In our case myosin and myoglobin were not expressed but less differentiated rhabdomyosarcoma can lack expression of these two antigens. It should be emphasised that desmin, expressed in our case, is the most sensitive marker for rhabdomyosarcoma. ${ }^{17}$

Rhabdomyoblastic differentiation may also represent part of the sarcomatous component of carcinosarcoma or malignant mixed tumour of the gall bladder. ${ }^{18}$ Twenty four cases of carcinosarcoma of the gall bladder had been reported in the world publications by 1990, but only in three of them has 
rhabdomyosarcomatous differentiation of a mesenchymal component been reported. ${ }^{18-20}$ We do not believe that our case belongs to this group of tumours, as the ultrastructural examination failed to show any epithelial differentiation and the immunoperoxidase stains showed negative expression of all epithelial markers used.

1 Edmonson HA. Tumours of the gallbladder and extrahepatic bile ducts. In: Atlas of tumour pathology. Section 7. Washington, DC: Armed Forces Institute of pathology, 1967: 82-3.

2 Yasuma T, Yanaka M. Primary sarcoma of the gallbladder. Acta Pathol fpn 1971; 21: 285-304.

3 Willen $\mathrm{R}$, Willen $\mathrm{H}$. Primary sarcoma of the gallbladder: a light and electron microscopical study. Virchows Arch $A$ 1982; 396: 91-102.

$4 \mathrm{Hsu}$ SM, Raine L, Fanger H. Use of avidin-biotinperoxidase complex (ABC) in immuno-peroxidase techniques. A comparison between $\mathrm{ABC}$ and unlabelled antibody (PAP) procedures. F Histochem Cytochem 1981; 29: $577-80$.

5 Ben Rejeb A, Bouziani A, Khelil A. Le Rhabdomyosarcome embryonnaire de type alveolire de la vesicule biliaire. A propos d'une observation. Arch Anat Cytol Pathol 1987; 35: 5-8.

6 Aldabagh SM, Shibata CS, Taxy JB. Rhabdomyosarcoma of the common bile duct in an adult. Arch Pathol Lab Med 1986; 110: 547-50.

7 Davis GL, Kissane JM, Ishak KG. Embryonal rhabdomyosarcoma (sarcoma botryoides) of the biliary tree: report of five cases and a review of the literature. Cancer 1969; 24: 333-42.
8 Akers DR, Needham ME. Sarcoma botryoides (Rhabdomyosarcoma) of the bile ducts with survival. $\mathcal{F}$ Pediatr Surg 1971; 6: 474-9.

9 Martinez-F LA, Haase GM, Koep LJ, Akers DR. Rhabdomyosarcoma of the biliary tree. The case for aggressive surgery. $\mathcal{I}$ Pediatr Surg 1982; 17: 508-11.

10 Mihara S, Matsumoto $H$, Tokunaga $F$, Yano $H$, Ota $M$ Yamashita $S$, et al. Botryoid Rhabdomyosarcoma of the gallbladder in a child. Cancer 1982; 49: 812-8.

11 Farinacci CJ, Fairchild JR, Sulak MH, Gilpatrick CW. Sarcoma botryoides of the common bile duct. Cancer Sarcoma botryoides

12 Horn RC, Yakovac WC, Kaye R, Koop CE. Rhabdomyosarcoma of the common bile duct (sarcoma botryoides). Cancer 1955; 8: 468-77.

13 Ruymann FB, Donaldson M, Lawrence W, Tefft M, Crist W. Extrahepatic biliary rhabdomyosarcoma [Abstract] Proc Am Assoc Cancer Res 1980; 21: 388.

14 Wilks $S$, Moxon W. Lectures on pathological anatomy. 2nd Ed. London: Longman, Brown, Green, Longmans, Robens and Co, 1875: 465-6.

15 Ruymann FB, Raney RB, Crist WM, Lawrence W, Lindber $\mathrm{RD}$, Soule EH. Rhabdomyosarcoma of the biliary tree in $\mathrm{RD}$, Soule EH. Rhabdomyosarcoma of the biliary tree in myosarcoma Study. Cancer 1985; 56: 575-81.

16 Isaacson C. Embryonal Rhabdomyosarcoma of the ampulla of Vater. Cancer 1978; 41: 365-8.

17 Tsokos $M$. The role of immunocytochemistry in the diagnosis of Rhabdomyosarcoma. Arch Pathol Lab Med 1986; 110: 776-8.

18 Von Kuster LC, Cohen C. Malignant mixed tumour of the gallbladder: report of two cases and review of the literature. Cancer 1982; 50: 1166-70.

19 Inoshita S, Iwashita A, Enjoji M. Carcinosarcoma of the gallbladder. Report of a case and review of the literature. Acta Pathol Fpn 1986; 36: 913-20.

20 Ichara T, Kawano H, Takahashi M, Yokota T, Uchino F Matsumoto N, et al. Carcinosarcoma of the gallbladder. A case report with immunohistochemical and ultrastructural studies. Cancer 1990; 66: 992-7. 